\section{Serotonin in the spotlight}

SIR - Since the early 1980s, many attempts have been made to use exposure to bright artificial light for the treatment of depression ${ }^{1}$. Indeed, for one type of depression, referred to as seasonal affective disorder (SAD) of the winter type, light therapy is now one of the preferred methods of treatment ${ }^{2}$. Because the external light-dark cycle is an important synchronizer of human circadian rhythmicity $^{3}$, and depression has been associated with disorders of temporal organization ${ }^{4,5}$, the therapeutic use of artificial bright light for SAD has been suggested to involve the effects of light on biological rhythms. However, the finding that artificial bright light can be effective in treating SAD even when presented in the middle of the day, when light exposure has no effects on circadian phase or day length ${ }^{6}$, is not consistent with the hypothesis that the antidepressive effects of light therapy are mediated by circadian phase changes and/or prolongation of the short days of winter.

The physiological basis for the effec-

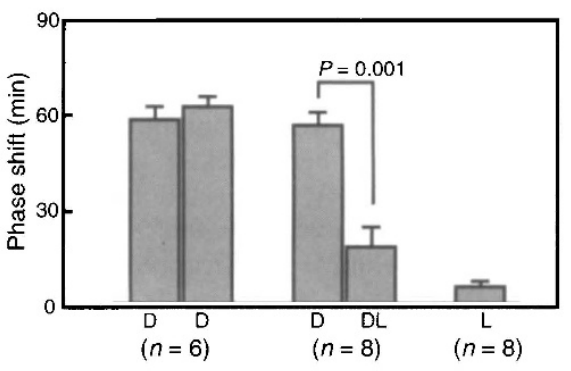

Treatment with 8 -OH-DPAT (D) $\left(1.0 \mathrm{mg} \mathrm{kg}^{-1}\right.$ interperitoneally) at circadian time 7 ( 5 hours before the onset of the subjective night), on two separate occasions, induced comparably large phase shifts in a group of six hamsters whose circadian rhythms were free-running in constant darkness (mean \pm s.e.m.; advances of $58 \pm 4$ and $62 \pm 3 \mathrm{~min}$ ). In contrast, the phase-shifting effects of 8-OH-DPAT in a similar group of eight animals were significantly attenuated when one of the treatments was combined with a 2-hour light pulse (DL) ( $56 \pm 4$ compared with $18 \pm 6$ min, $P \leqslant 0.001$ ). Consistent with the existence of a 'dead zone' for photic phase-resetting of the mammalian circadian pacemaker during the subjective day, exposure to the photic stimulus alone (L) (400-600 lux white fluorescent light) had no effect on circadian phase in an additional group of eight animals ( $5 \pm 2 \mathrm{~min}$ ).

\section{Scientific Correspondence}

Scientific Correspondence is intended to provide a forum in which readers may raise points of a scientific character. Priority will be given to letters of fewer than 500 words and 10 references. tive use of light in the treatment of SAD remains unknown. Here we report new data from rodents which raise the possibility that light can significantly modify the processing of serotonergic signals by the mammalian brain.

The selective serotonin re-uptake inhibitors represent one of the most commonly used pharmacological agents for the treatment of depressive illness ${ }^{7}$. In addition to the role of serotonergic mechanisms in the pathogenesis of depression, several animal studies reveal the involvement of serotonin (5-HT) in the control of mammalian circadian rhythmicity. Together with direct and indirect photic input from the retina, the circadian pacemaker in the suprachiasmatic nucleus of the hypothalamus receives serotonergic projections from the midbrain raphe nuclei.

Further, recent studies have demonstrated the ability of several serotonergic stimuli to: (1) advance the phase of the circadian clock when administered during the subjective day ${ }^{8}$; and (2) block the phase-shifting effects of light on circadian rhythmicity during the subjective night (a time when the serotonin stimuli alone have no effect on circadian phase $)^{9}$. The existence of these anatomical and functional connections between retinal and raphe projections to the rodent circadian system provides an excellent model in which to study the effects of light on serotonergic neurotransmission in the mammalian brain.

Here, we used the well-defined ability of the serotonin subtype $5-\mathrm{HT}_{1 \mathrm{~A}}$ agonist, 8-hydroxy-dipropylaminotetralin (8-OHDPAT), to induce phase advances in the circadian rhythm of locomotor activity in golden hamsters (Mesocricetus auratus) during the subjective day. The effects of this stimulus were examined in the same group of animals on two separate occasions, with and without concomitant exposure to a 2-hour light pulse. Consistent with the existence of a 'dead zone' for photic phase-resetting of the circadian pacemaker during most of the subjective day, exposure to the photic stimulus alone had no effect on circadian phase in control animals. However, this intervention considerably attenuated or completely blocked the phase-resetting properties of the $5-\mathrm{HT}_{1 \mathrm{~A}}$ agonist in the experimental group of hamsters (see figure).

The described ability of light to modify the processing of serotonergic stimuli in the circadian system suggests new mechanisms that may underlie the beneficial effects of artificial light therapy in circadian timing and mood disorders. On the one hand, it indicates that, in the presence of multiple time-giving cues, light exposure can play an important role in circadian phase control even during the 'dead zone' for photic resetting of the circadian pacemaker. On the other hand, it raises the possibility that photic stimulation may, in a similar fashion, alter the functional status of serotonergic pathways outside our circadian model framework - changes which could have important implications for the use of light as a 'drug' to alter neurochemical activity in the brain.

Plamen D. Penev

Phyllis C. Zee

Fred W. Turek

Center for Circadian Biology and Medicine, Northwestern University,

Evanston,

IIIinois 60208, USA

1. Thalen, B. E. et al. Acta Psychiatr. Scand. 91, 352-360 (1995).

2. Diagnostic and Statistical Manual of Mental Disorders 4th edn 389 (Am. Psychiatric Assoc., Washington DC, 1994).

3. Boivin, D. B., Duffy, J. F., Kronauer, R. E. \& Czeisler, C. A. Nature 379, 540-542 (1996)

4. Lewy, A. J., Sack, R. L., Miller, L. S. \& Hoban, T. M. Science 235, 352-354 (1986).

5. Van Cauter, E. \& Turek, F. W. Perspect. Biol. Med. 29 , 510-519 (1986)

6. Wirz-Justice, A. et al. Arch. Gen. Psychiatry 50, 929-937 (1993).

7. Andrews, J. M. \& Nemeroff, C. B. Am. J. Med. 97 , 24S-32S (1994).

8. Lovenberg, T. W. et at. Neuron 11, 449-458 (1993).

9. Rea, M. A., Glass, J. D. \& Colwell, C. S. J. Neurosci. 14, 3635-3642 (1994).

\section{p53 and treatment of bladder cancer}

SIR - The report by Waldman et al. ${ }^{1}$ that tumours with alterations in the gene p53 may exhibit increased sensitivity to DNA-damaging agents is in significant contrast to the prevailing view that p53-altered cells are more resistant to chemotherapy because they are less able to undergo apoptosis (programmed cell death $)^{2}$. This view is supported by clinical studies indicating that p53-altered tumours are more resistant to chemotherapy than p53 wild-type tumours ${ }^{3}$. The response of p53-altered tumours to chemotherapy has profound implications for the selection of patients that would most benefit from such treatment. Here we show that p53-altered bladder cancers may show increased sensitivity to chemotherapy that includes DNA-damaging agents.

We have previously shown that patients with transitional cell carcinoma (TCC) of the bladder who demonstrate p53 alterations have a significantly increased rate of developing tumour metastases and dying of the disease, compared with patients with TCC who have no evidence of p53 alterations ${ }^{4}$. We have also shown, 